\title{
Prepulse inhibition and P50 suppression: Commonalities and dissociations
}

\author{
Bob Oranje $^{\mathrm{a}, *}$, Mark A. Geyer ${ }^{\mathrm{c}}$, Koen B.E. Bocker ${ }^{\mathrm{b}}$, \\ J. Leon Kenemans ${ }^{\text {b }}$, Marinus N. Verbaten ${ }^{\text {b }}$ \\ a Center for Neuropsychiatric Schizophrenia Research, Department of Psychiatry, Bispebjerg University Hospital, Bispebjerg Bakke 23, \\ 2400 Copenhagen NV, Denmark \\ ${ }^{\mathrm{b}}$ Department of Psychopharmacology, Faculty of Pharmaceutical Sciences, Rudolf Magnus Institute for Neurosciences, \\ Utrecht University, The Netherlands \\ ${ }^{c}$ Department of Psychiatry, University of California, San Diego, CA, United States
}

Received 15 October 2004; received in revised form 27 September 2005; accepted 8 November 2005

\begin{abstract}
Patients with schizophrenia exhibit reduced levels of both prepulse inhibition of the startle reflex (PPI) and condition-test suppression of the P50 event-related potential. This study investigated the extent to which PPI and P50 suppression, which exhibit similar parametric sensitivities, are intrinsically auditory phenomena or can be induced cross-modally, and reflect common or distinct neural mechanisms of inhibition. PPI, N100, and P50 were assessed in 20 healthy male volunteers, using auditory test probes and both visual and auditory lead stimuli, separated by 100- or 500-ms interstimulus intervals (ISIs). PPI was found in the auditory-lead condition across the complete group, and with visual-lead stimuli in approximately half of the subjects. Intra-modal auditory PPI was significantly higher with the 100-ms ISI than with the 500-ms ISI. P50 suppression was found only with the 500-ms ISI, with no difference between the auditory and visual conditions. Source analyses revealed that suppression was associated with frontal cortical activity. N100 suppression was found only in the auditory condition, with no difference between 100- and 500-ms ISIs. Although both phenomena are considered to provide operational measures of gating, PPI and P50 suppression are differentially sensitive to ISI and therefore reflect partly different neural mechanisms. They are not intrinsically auditory phenomena, and both appear to involve frontal cortical activity. In contrast, N100 suppression is most likely based on refractory mechanisms intrinsic to the auditory system.
\end{abstract}

(C) 2005 Elsevier Ireland Ltd. All rights reserved.

Keywords: PPI; P50 suppression; Cross-modal; Source localization; Sensory gating; N100

\section{Introduction}

The ability to inhibit responses to incoming sensory information is an important feature of a healthy individual. Following extensive observation of changes in perceptual awareness in patients with schizophrenia,

\footnotetext{
* Corresponding author. Tel.: +45 35312391.

E-mail address: B.Oranje@cnsr.dk (B. Oranje).
}

McGhie and Chapman (1961) postulated that an organism needs an internal mechanism to select from diverse sensory input the information that is needed to allow it to function efficiently, a process which has come to be termed sensory or sensorimotor gating. Patients with schizophrenia show deficits in gating that subsequently might lead to a state of flooding (McGhie and Chapman, 1961; Venables, 1964) and, ultimately, to the formation of psychoses (Braff and Geyer, 1990; Freed- 
man et al., 1991). Over time, two paradigms have been developed to operationally quantify the amount of sensory or sensorimotor gating.

In the P50 suppression paradigm, two identical auditory stimuli are presented in close temporal proximity to each other (usually $500 \mathrm{~ms}$ ), in a condition-test paradigm. The amplitude of the P50 evoked potential to the first, or conditioning, stimulus is larger than the response to the second, or testing stimulus. P50 suppression is a measure of sensory gating, because an event related potential (ERP) rather than a motor response is the dependent measure. Adler et al. (1982) found that (unmedicated) patients with schizophrenia exhibited less P50 suppression than healthy control subjects, a finding that has been replicated in numerous subsequent studies (e.g. Nagamoto et al., 1989, 1991; Ward et al., 1996; Clementz et al., 1997).

In the prepulse inhibition of startle (PPI) paradigm, the measure is the motor startle reflex elicited by a sudden and strong sensory stimulus, usually a loud noise. This reflex can be reduced by a weaker stimulus (e.g., a soft sound), which precedes the startleeliciting stimulus (Graham, 1975). Because the first, or prepulse, stimulus reduces the effect of the second, or startle-eliciting, stimulus, percentage PPI provides a measure of the amount of sensorimotor gating. Braff et al. (1978) reported a reduction of PPI in patients with schizophrenia compared with healthy control subjects, a finding that, like the P50 suppression paradigm, has been replicated in numerous studies (e.g.: Braff et al., 1992; see review in Braff et al., 2001), although not in all (Ford et al., 1999; Wynn et al., 2004; Kumari et al., 2004), probably due to methodological differences, e.g., no background noise or gender-related issues.

Although both paradigms are thought to measure gating of incoming information, they are based on different physiological phenomena: P50 suppression is measured by means of electroencephalography (EEG), while PPI is measured by means of electromyography (EMG). The limited evidence available indicates that PPI and P50 suppression are only weakly related in either healthy volunteers or in patients with schizophrenia (Schwarzkopf et al., 1993; Oranje et al., 1999; Cadenhead et al., 2002).

Despite this weak relation between the read-out measures in the two paradigms, commonality might still exist for the underlying neural mechanisms. For example, both PPI and P50 suppression could involve common forebrain mechanisms that serve to regulate cortical and brainstem responsivity. The primary neural control of the startle reflex involves brain struc- tures at, or below, the level of the mesencephalon: the auditory nerve, the ventral cochlear nucleus, the dorsal nucleus of the lateral lemniscus, the caudal pontine reticular nucleus, spinal interneurons and spinal motor neurons (Davis et al., 1982). Modulation of the startle reflex however (apart from the above-mentioned PPI, this includes phenomena such as habituation, sensitization and fear potentiation), has been suggested to involve a number of brain structures located up to the forebrain (for reviews: Swerdlow et al., 1992, 2001; Koch, 1999). Unlike the startle reflex in the PPI paradigm, P50 suppression does not have a substrate that is easily identified across animal species. Therefore, a limited amount of literature is available on the neural substrates that are involved. Bickford-Wimer et al. (1990) showed that the pattern of diminished response to the second of paired stimuli is found in activity recorded from the CA3 region of the hippocampus in anaesthetized rats (N40 wave). Furthermore, in a review, Adler et al. (1998) propose a model of P50 suppression in which the first stimulus induces a 50-ms burst of activity in the pyramidal cells of the CA3 region of the hippocampus, which is suggested to be the source of the P50 wave as measured on the scalp surface. The second stimulus can only excite a modest amount of pyramidal cells due to recurrent excitation elicited by the first stimulus (Adler et al., 1998).

One approach to the issue of common underlying mechanisms is to compare the sensitivity of both phenomena to various experimental manipulations. An obvious choice in this respect is the interstimulus interval (ISI) between lead and test stimuli. For PPI following auditory prepulses, it is well established that the optimal ISI is about $100 \mathrm{~ms}$, and that PPI rapidly disappears when the ISI is extended by only a few hundreds of milliseconds (Braff et al., 1978; Ison and Hoffman, 1983; Dawson et al., 1995). P50 suppression is commonly observed with a 500-ms ISI. However, it is less clear how it responds to reductions of the ISI (e.g., to $100 \mathrm{~ms}$ ) (Nagamoto et al., 1989, 1991). A second possibility is to manipulate the modality of the stimuli. Neither PPI nor the schizophrenia-linked deficits in PPI are limited to the auditory system, and both are evident with either intramodal or cross-modal stimulus pairs (Ison and Hoffman, 1983; Braff et al., 1992). On the other hand, it is still largely unclear whether P50 suppression involves only dedicated auditory circuits, or whether modality-nonspecific structures are involved as well. In the latter case, it is conceivable that P50 suppression can also be induced with lead stimuli in a 
different modality, whereas this is highly unlikely in the former scenario. A related question concerns the localization of the evoked activity that is actually suppressed in P50 suppression: Is it exclusively auditory cortex, or are frontal or perhaps even medialtemporal areas involved?

The present study was designed to address these questions by manipulating ISI and lead-stimulus modality, and by source analysis (BESA) of the P50. A special feature of the design was that the traditional PPI and P50 suppression paradigms were integrated into one test session, in which the first (conditioning) stimulus of the P50 trials was also used as the prepulse in the PPI trials. This design excluded the possibility of differences between the paradigms that are due to irrelevant, nonspecific factors (e.g. some tonic sensitization by loud stimuli, which are presented in the PPI but not in the P50 paradigm). The suppression of a later ERP component, i.e. the $\mathrm{N} 100$, in the P50 trials of the paradigm was studied to investigate whether responses to the testing stimulus are blocked completely or only partially from further processing, as a result of the conditioning stimulus.

\section{Methods}

\subsection{Subjects}

Twenty healthy male volunteers were recruited through university newspaper advertisements. Only physically healthy subjects without a personal or family history in first-degree relatives of psychiatric illness were included. The study was approved by the Human Ethics Board of the University Medical Center Utrecht, taking into account the statements for human research from Helsinki (Amendment of South Edinburgh from 2001). After written and oral information had been given, written informed consent was obtained from all subjects before enrollment in the study. Subsequently, they were interviewed using the Comprehensive Assessment of Symptoms and History (CASH) (Andreasen et al., 1992) and the Schedule for Affective Disorders and Schizophrenia, Lifetime version (SADS-L) (Endicott and Spitzer, 1978) to ascertain absence of psychiatric illnesses.

Of the 20 subjects initially included in the study, three subjects had to be excluded from P50 suppression analysis for not showing an identifiable P50 waveform to the auditory conditioning stimulus. This resulted in 20 subjects in the PPI paradigm (mean age: 24.9, S.D. $=3.62)$ and 17 subjects in the P50 suppression paradigm (mean age: 24.6, S.D. $=3.63$ ).

\subsection{Experimental design and tasks}

Subjects were tested in a sound and electrically shielded experimental cabin, where they were seated comfortably in a reclining chair. During a test session, two versions of the same task were presented: an auditory and a visual variant, of which the sequence was balanced across subjects.

In the auditory variant, three auditory stimuli (white noise) were presented: $\mathrm{S} 1=\mathrm{S} 2(1.5 \mathrm{~ms}, 80 \mathrm{~dB})$ and $\mathrm{S} 3$ $(40 \mathrm{~ms}, 110 \mathrm{~dB})$. In addition, two ISIs were used, i.e. $100 \mathrm{~ms}$ and $500 \mathrm{~ms}$, while the intertrial intervals were randomized between 5 and $10 \mathrm{~s}$. Randomized across the task, six types of trials were presented: S1 was presented 75 times, S3 15 times, the combination S1-S2 was presented 75 times for ISI $100 \mathrm{~ms}$ and 75 times for ISI $500 \mathrm{~ms}$, the combination $\mathrm{S} 1-\mathrm{S} 3$ was presented 15 times for ISI $100 \mathrm{~ms}$ and 15 times for ISI $500 \mathrm{~ms}$, totaling up to a number of 270 trials for this task which lasted $30 \mathrm{~min}$. The intensity, duration and presentation of the stimuli were chosen in such a way that the trials closely resemble the P50 suppression and PPI paradigms commonly used in literature (i.e. S1 represents the conditioning stimulus in the P50 trials, and prepulse in the PPI trials, S2 represents the testing stimulus in the P50 suppression trials, and S3 represents the startleeliciting stimulus in the PPI trials).

In the visual version of the paradigm, stimulus S1 was replaced by a dot of white light $(r=15 \mathrm{~cm})$, appearing on a monitor at $90 \mathrm{~cm}$ distance of the subject. All auditory stimuli were gated almost instantaneously (rise/ fall time $<0.1 \mathrm{~ms}$ ) and presented binaurally through stereo insert earphones (Eartone ABR). Please note that in both the auditory and visual versions of the task S1 serves as both the conditioning stimulus in the P50 trials as well as the prepulse in the PPI trials. During the experimental blocks, a continuous $70-\mathrm{dB}$ white noise acted as a background noise. Furthermore, during a test session, subjects were asked to fix their gaze on a fixation point presented on a monitor. To prevent drowsiness, both versions of the tasks were divided into two blocks of equal length. In between blocks and tasks, subjects were briefly asked whether they still felt fit, before continuing with the second block or task. At the end of the experiment, the positions of the electrodes on the scalp were measured with a 3D digitizer (Polhemus).

\subsection{Signal recording}

Electroencephalographic (EEG) recordings were made with an electrocap (tin electrodes) from 62 scalp locations (10-20 system). However, for assessment of 
the P50 and N100 ERP, only data from electrode Cz were analyzed (i.e. where the maximum activity for the P50 ERP was to be expected (Clementz et al., 1998)). The left mastoid was used as a reference. Horizontal electro-oculographic (EOG) recordings were made from tin electrodes placed to the outer canthus of each eye. Similarly, vertical EOG was recorded from tin electrodes placed infra-orbital and supra-orbital to the left eye. The electro-myographic (EMG) activity of both the right and left orbicularis inferior muscles was recorded bipolarly: one electrode was located on the medial part of the muscle, the second one was located 1 $\mathrm{cm}$ exterior, in the direction of the outer canthus of the eye. For all signal recordings, a ground electrode was attached to the middle of the forehead. Impedance was kept below $5 \mathrm{k} \Omega$. All EEG, EOG and EMG signals were recorded with an on-line filter (low-pass) set at $250 \mathrm{~Hz}$. Sampling started as soon as an experimental block began, and lasted until the end of it (continuous recording). All signals were digitized on-line by a computer, at a rate of $1 \mathrm{kHz}$.

\subsection{Data processing}

Post hoc processing of the data started with epoching the recorded signals from $50 \mathrm{~ms}$ prestimulus to $350 \mathrm{~ms}$ poststimulus. In the case of P50 and N100 assessment (stimuli S1 and the combination S1-S2), the data were filtered (low-pass) at $40 \mathrm{~Hz}$, after which the data were baseline ( -50 to 0$)$ corrected.

\section{5. $E M G$}

Assessment of the maximum (absolute) peak amplitude and PPI quantification took place within a window of 20-90 ms after stimulus onset, using a computerized algorithm for peak detection. Prepulse inhibition was computed as the percentage reduction of the amplitude of pulse trials preceded by a prepulse over pulse alone trials, i.e. PPI $=100(1-\mathrm{pp} / p)$, where $\mathrm{pp}$ indicates the mean amplitude to pulse trials preceded by a prepulse (S3 preceded by $\mathrm{S} 1$, either auditory or visual) and $p$, the mean amplitude over pulse alone trials (S3 presented alone).

\section{6. $E E G$}

First, all epochs containing artifacts (saturation of the A/D converter ("clipping") or absolute amplitudes larger than $100 \mu \mathrm{V}$ ) were removed from the database (less than 5\%). This step was followed by the correction of the EEG for eye artifacts (eye-blinks and move- ments), by subtracting horizontal and vertical EOG from EEG epochs by means of a regression in the time domain (Kenemans et al., 1991). To eliminate overlap of the ERPs in the assessments of stimulus $\mathrm{S} 2$, the averaged activity of the S1 stimulus presented alone was subtracted from the averaged S1-S2 activity in the 100-ms ISI. Following this procedure, the N100 elicited by both the auditory and the visual S1 stimuli presented alone was scored between 50 and $200 \mathrm{~ms}$. The P50 waves were identified and scored as described by Nagamoto et al. (1989): P50 peaks elicited by the auditory S1 stimulus presented alone were identified as the greatest positivity in a window from 40 to $90 \mathrm{~ms}$. If more than one peak was identified, the later one was selected. The amplitude was assessed as the difference between this peak and the preceding trough, the latency was assessed as the time from the onset of the conditioning stimulus to the maximum amplitude of this peak. The P50 peak elicited by the second (testing) stimulus (S2, which was always a sound) was assessed accordingly, with a further constraint that its peak-latency was in a window of the P50 latency evoked by the conditioning stimulus $\pm 10 \mathrm{~ms}$. Two raters were used to identify the P50 wave; the interrater reliability was higher than 0.95. P50 suppression was defined as the ratio $T / C$, where $T$ represents the mean P50 amplitude to the testing stimuli, split in testing stimuli preceded by either auditory or visual conditioning stimuli, while $C$ represents the mean P50 amplitude to the auditory conditioning stimuli only. Similar to P50 suppression, the N100 suppression was defined as the ratio $T / C$, in which $\mathrm{T}$ represents the N100 amplitude to the testing stimulus (again, split in the auditory and visual modalities), and $C$ the N100 amplitude to the auditory conditioning stimulus.

\subsection{Dipole analysis}

Following re-reference of the data to the average reference, dipole analyses using BESA software (Scherg, 1990; Scherg and Picton, 1991) were performed on the grand-average ERPs for the auditory S1 stimuli presented alone, and that for the combination $\mathrm{S} 1-\mathrm{S} 2$ for the ISI $500 \mathrm{~ms}$. Digitized electrode locations were averaged over subjects, projected on a leastsquares fitted sphere, which was rotated with respect to nasion and mastoid locations. In BESA, the default-3 shell model was used. Modeling at the exact moment of maximum P50 amplitude or from the start of P50 appearance to the end each resulted in unstable fits. Modeling the interval that covered both the P50 ERP and the peak of the N100 (i.e. 56-112 ms) yielded stable results. 
This model was inspected for its fit at the peak latency of P50, in both the S1 and S2 ERP. Before modeling, the data were baseline corrected for the trough relative to which the P50 amplitude was measured, to assure a model comparable to the assessment of the P50 amplitude. In dipole modeling, the cost function was defined by the amount of residual variance (a measure that indicates the fit of the data on the model). Solutions had to be robust with regard to the starting values of the dipole. Since in addition to auditory induced P50 suppression, visually induced P50 suppression was also found, the grand average ERP to the visual S1 was also modeled in the P50 latency region (56-123 ms).

\subsection{Data analysis}

First, PPI data were examined for differences between startle assessment at the left and right orbicularis occuli muscles. Because no differences were found, these data were collapsed. Second, P50 suppression (S2 amplitude divided by S1 amplitude) and percentage PPI were examined through analysis of variance (ANOVA) with repeated measures with factors "modality" (auditory versus visual lead stimulus) and "ISI" (ISI $100 \mathrm{~ms}$ versus $500 \mathrm{~ms}$ ). Student's $t$-tests were used for further exploration of the significant effects revealed in the ANOVAs. Since it seems irrelevant to compare the response to a visual stimulus with that evoked by an auditory stimulus, only the P50 amplitude to the auditory conditioning stimulus was used to calculate P50 suppression, since all of the testing stimuli were in the auditory modality. For analysis of N100 suppression, the same procedure was used as in the analysis of P50 suppression. Differences between the visual and auditory S1 dipole models of the P50 ERP were evaluated statistically by optimizing both models for each individual subject and entering the resulting three-dimensional individual coordinates in a two-way ANOVA.

\section{Results}

\section{1. $E E G$}

In the P50 paradigm, a main effect of ISI $[F(1,16)=10.84 ; P<0.005]$ was found. There were no differences between P50 suppression as a result of visual versus auditory lead stimuli. Further testing revealed significant P50 suppression in the 500-ms ISI condition only, for both the auditory $(t=5.03$, $P<0.001)$ and visual modalities $(t=2.19, P<0.05)$ (Figs. 1 and 2, Table 1). Besides a P100, a P50 ERP was found following visual lead stimuli, although it appeared to be smaller than in the case of the auditory lead stimuli (Table 1 and Fig. 3).

In contrast to P50 suppression, the analysis of the N100 showed a main effect of modality $[F(1,19)=8.29$;

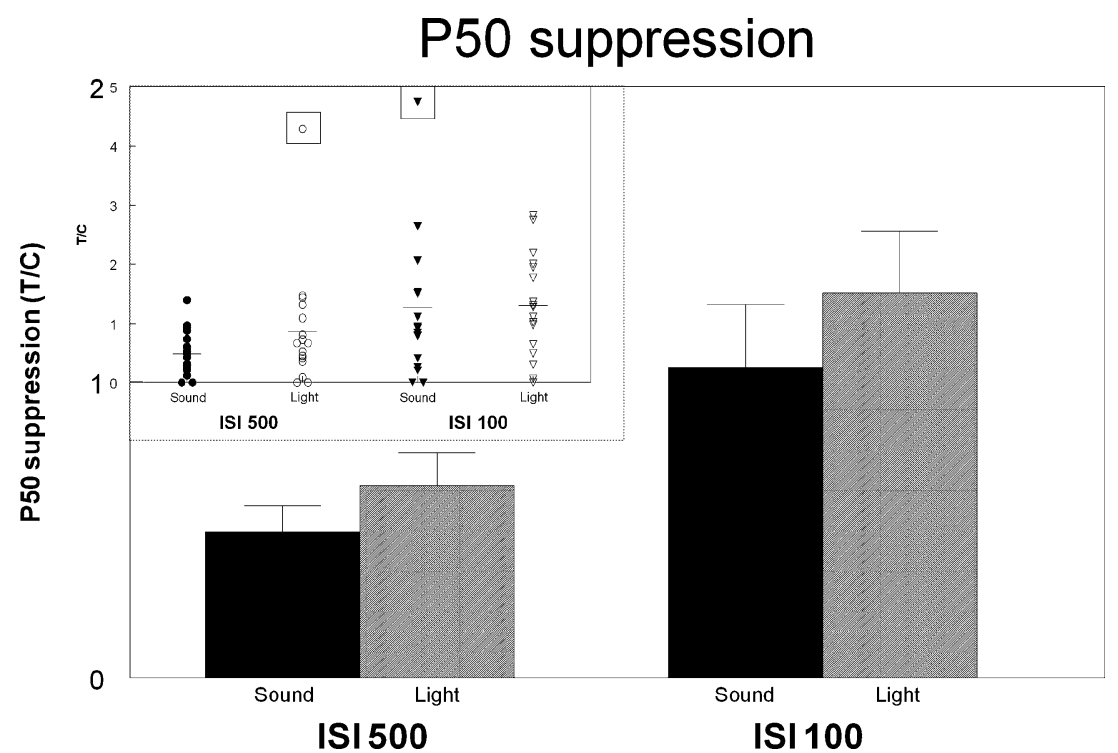

Fig. 1. P50 suppression ( $\pm \mathrm{SEM}$ ), as expressed by $T / C: C=\mathrm{P} 50$ amplitude to auditory conditioning stimulus, $T=\mathrm{P} 50$ amplitude to each of the four different testing stimuli (two different interstimulus intervals (ISI 100 and $500 \mathrm{~ms}$ ) and two different modalities (auditory and visual lead stimulus)). In the upper left corner a boxplot of the data is displayed, indicating two outlying values (marked in boxes). No changes occur in the statistical significance on exclusion of these two values, although the $P$-values decrease slightly. The two values were excluded from the bar figure. The difference in P50 suppression between the ISI of $500 \mathrm{~ms}$ and $100 \mathrm{~ms}$ is significant, but between the auditory and visual modalities, it is not. 

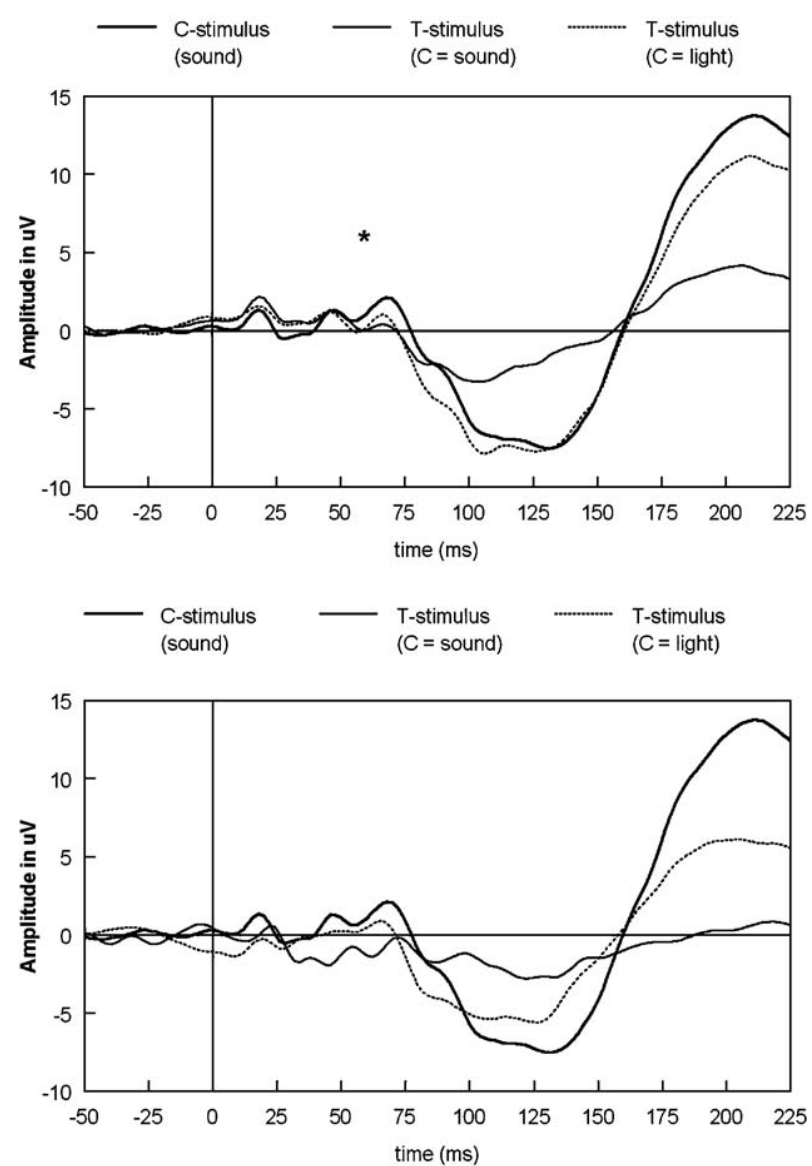

Fig. 2. Grand average data of lead CZ of both the 500-ms (above) and 100-ms (below) ISIs. Displayed are the responses to the auditory conditioning stimulus (thick line in both figures) and the different kind of testing $(T)$ stimuli: following the auditory lead stimulus (thin solid line) and following the visual lead stimulus (broken line). Scoring of the individual data showed significant P50 suppression of the testing stimulus compared with the auditory lead stimulus in the 500-ms ISI only $\left({ }^{*} P<0.05\right)$, regardless of the modality of the lead stimulus (see also Fig. 1). The data for the testing stimuli in the ISI-100 condition are corrected for overlap of the conditioning stimulus (see Section 2.6).

$P<0.01]$, but no effect of ISI. Further testing on modality revealed N100 suppression in the auditory modality only, for both the 100-ms ISI $(t=3.77$, $P<0.001)$ and the 500-ms ISI $(t=6.36, P<0.001)$ (Fig. 4).

\section{2. $E M G$}

In the PPI paradigm, significant effects of modality $[F(1,19)=43.35 ; P<0.001]$ and ISI $[F(1,19)=22.53$; $P<0.001]$ were found, indicating higher levels of

Table 1

P50 amplitude (in $\mu \mathrm{V} \pm \mathrm{SEM}$ ) for the two different interstimulus intervals (ISI 100 and $500 \mathrm{~ms}$ ) and two different modalities (auditory and visual lead stimulus)

\begin{tabular}{llll}
\hline Modality & $C$-stimulus & $T$-stimulus (ISI 500) & $T$-stimulus (ISI 100) \\
\hline Sound & $3.50(0.58)$ & $1.74(0.54)^{*}$ & $3.25(0.67)$ \\
Light (total) & $2.30(0.43)$ & $2.22(0.49)^{*}$ & $3.71(0.63)$ \\
$\quad$ PPI responders $(n=9)$ & $3.23(0.44)$ & - & - \\
PPI non responders $(n=11)$ & $1.75(0.44)^{* *}$ & - & - \\
\hline
\end{tabular}

The P50 amplitude in the visual modality is split into a group of subjects who did show PPI to light as a prepulse (PPI $>10 \%)$, and a group of subjects who did not $(\mathrm{PPI}<\%)$. Please note that only the P50 amplitude to the auditory conditioning stimulus $(3.50 \mu \mathrm{V})$ was used to assess P50 suppression of the four different testing stimuli.

* Indicates a significant $(P<0.05)$ reduction in P50 amplitude compared to the auditory $C$-stimulus.

** Indicates a significant $(P<0.05)$ difference in P50 amplitude to the visual lead stimulus between PPI responders and PPI non-responders 


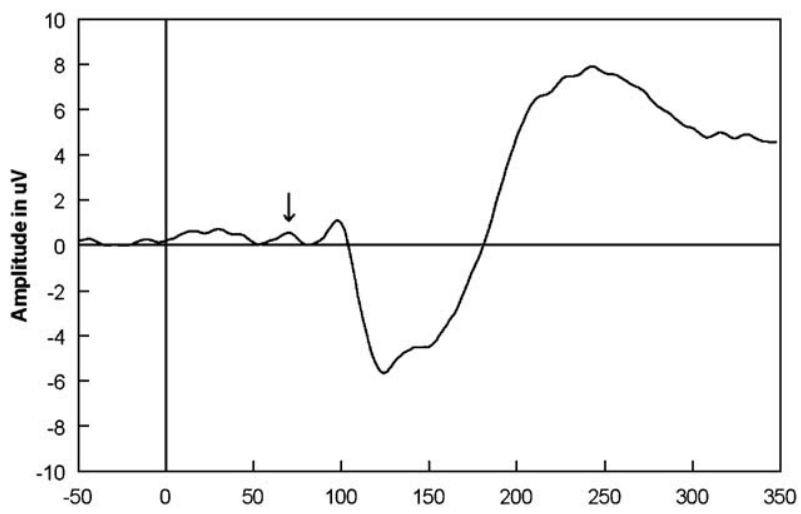

Fig. 3. Grand average data of electrode $\mathrm{CZ}$ for the visual conditioning stimulus only. The arrow indicates activity in the same interval as that of the P50 wave to the auditory conditioning stimulus. Note, however, that this activity is relatively small in comparison with that found in the auditory modality.

percent PPI with auditory prepulses than with visual prepulses and higher percent PPI in the $100-\mathrm{ms}$ interval than in the 500-ms interval, respectively. Furthermore, a significant interaction between the effects of prepulse modality and ISI $[F(1,19)=4.34$; $P<0.05]$ was found, indicating a larger decrease in percent PPI from the ISI $=100 \mathrm{~ms}$ to the ISI $=500 \mathrm{~ms}$ condition with the auditory prepulse than with the visual prepulse (Fig. 5). Further testing revealed significant PPI following the auditory prepulse only (ISI
100 ms: $t=4.94 ; P<0.001$, ISI $500 \mathrm{~ms}: t=3.68$, $P<0.005)$.

\subsection{Correlations between PPI and P50 suppression}

No significant correlations were found between parameters of PPI and P50 suppression, neither in the visual nor in the auditory modality. Nevertheless, although no significant overall PPI was found with a visual prepulse, roughly half (9) of the subjects seemed

N100 suppression

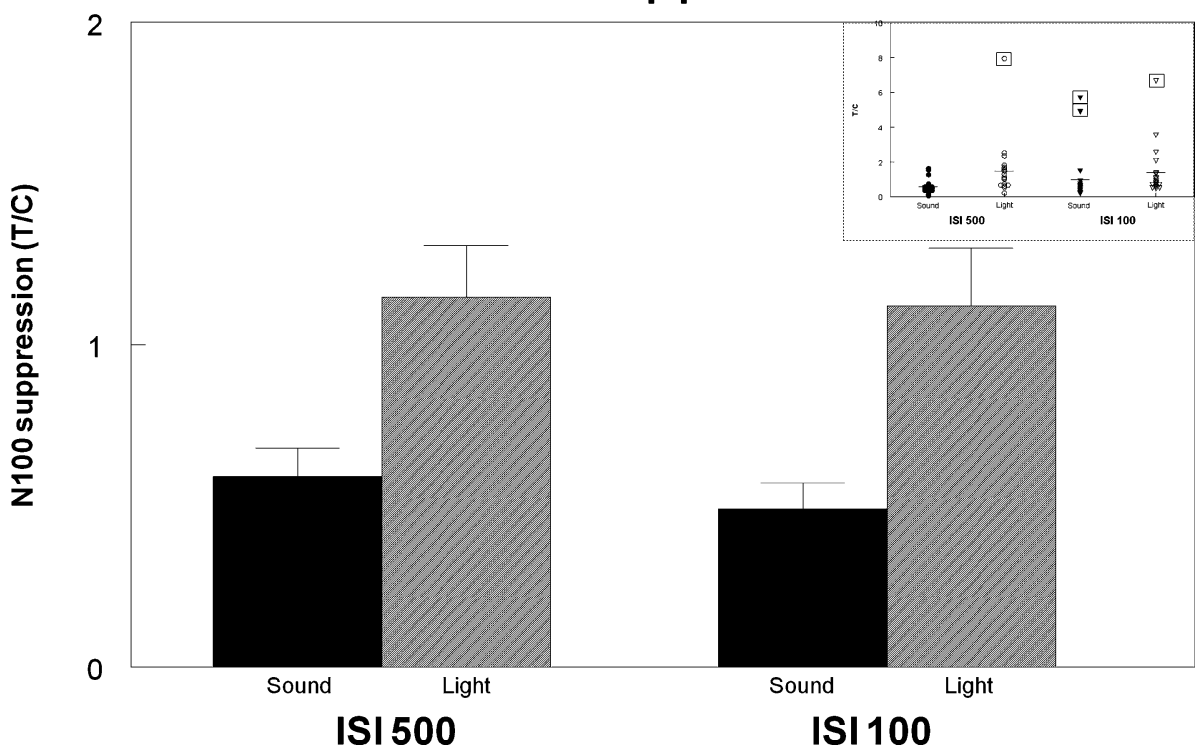

Fig. 4. N100 suppression, as expressed by $T / C(C=\mathrm{N} 100$ to auditory stimulus only), for the two different interstimulus intervals (ISI 100 and 500 $\mathrm{ms}$ ) and two different modalities (prepulse auditory and visual). In the upper right corner a boxplot of the data is displayed, indicating four outlying values (marked in boxes). No changes occur in the statistical analysis on excluding these four values. The outlying values were excluded from the bar figure. The difference in N100 suppression between the auditory and visual modalities is significant (with N100 suppression auditory >N100 suppression visual); the difference between ISI $500 \mathrm{~ms}$ and $100 \mathrm{~ms}$ is not. Moreover, the difference between the N100 $C$ and $T$ amplitude is only significant for both auditory ISIs, and not for the visual ISIs. 


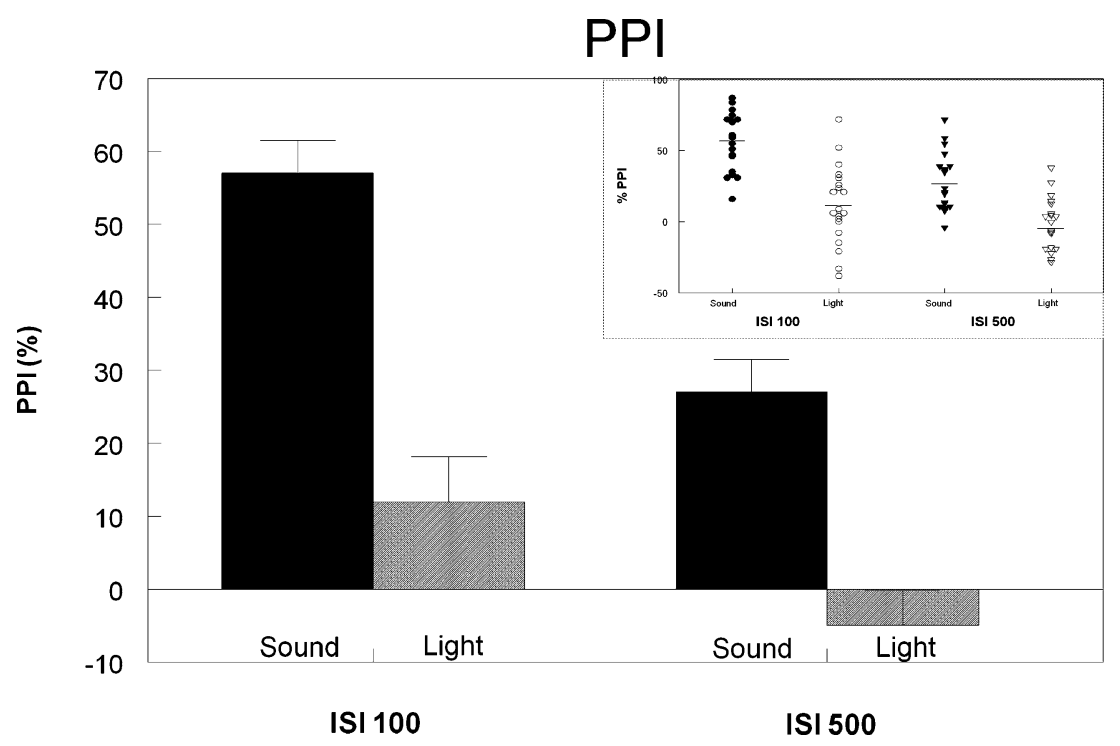

Fig. 5. Percentage prepulse inhibition $( \pm$ SEM) of the startle reflex (PPI) for the two different interstimulus intervals (ISI 100 and $500 \mathrm{~ms})$ and two different modalities (auditory and visual lead stimulus). In the upper right corner a boxplot of the data is displayed. Both the differences in PPI between the sound and light modality (with PPI sound > PPI light) and between the ISI $100 \mathrm{~ms}$ and ISI 500 ms condition (with PPI $100 \mathrm{~ms}>$ PPI 500 ms) are significant.

to respond to the visual prepulse by showing PPI, while the other half (11) did not (see boxplot in Fig. 5). Therefore, the PPI data were divided post hoc into a group of responders (PPI $>10 \%$ ) and a group of nonresponders $(\mathrm{PPI}<10 \%)$ to visual prepulses. The group of responders had a significantly higher P50 amplitude to the visual stimulus $(t=2.33 ; P<0.05)$ (Table 1). To investigate whether a similar phenomenon (a higher percentage PPI in case of a higher P50 amplitude to the prepulse) was also present in the auditory modality, the subjects were again split into two groups, only this time on the basis of their P50 amplitude to the auditory prepulse (amplitudes above or below $1.75 \mu \mathrm{V}$ ); the group with P50 amplitudes above $1.75 \mu \mathrm{V}$ showed more PPI than the group with P50 amplitudes below $1.75 \mu \mathrm{V}$ (\%PPI 60.8 and 51.9, respectively), although this difference did not reach statistical significance.

\subsection{Dipole analysis}

\subsubsection{P50}

Dipole analysis of the grand average P50 ERPs elicited by $\mathrm{S} 1$ alone resulted in a three-source model for the auditory (fitted on the interval $56-112 \mathrm{~ms}$ poststimulus) and a four-source model for the visual modality (fitted on the interval $56-123 \mathrm{~ms}$ poststimulus), with residual variances of $3.29 \%$ and $3.64 \%$, respectively. The auditory and visual models appear to have a frontal source in common (Fig. 6). Analysis of the individual three-dimensional coordinates of this source revealed no significant differences between the

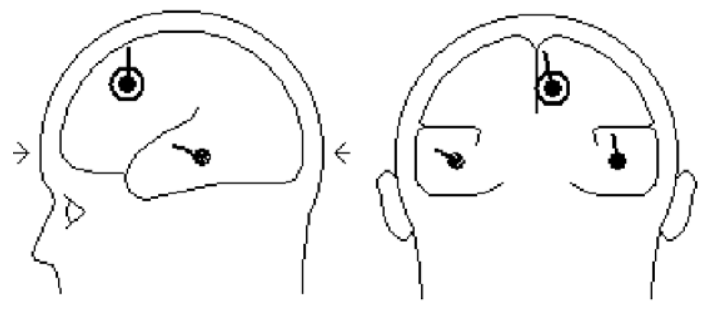

Auditory modality

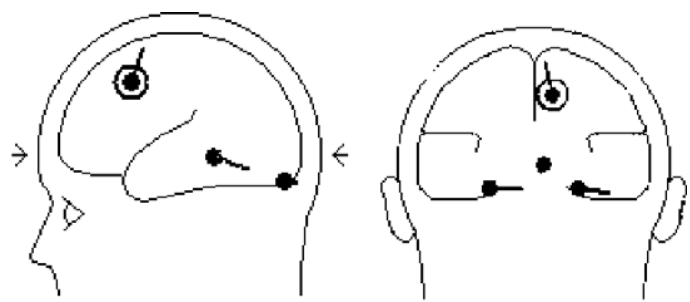

Visual modality

Fig. 6. BESA source localization modeling of the P50 ERP elicited by the conditioning stimulus for both the auditory and visual modality, based on a time interval of 56-112 $\mathrm{ms}$ in the auditory modality and 56-123 ms in the visual modality. The models seem to have one source in common (encircled). 
Sources N100
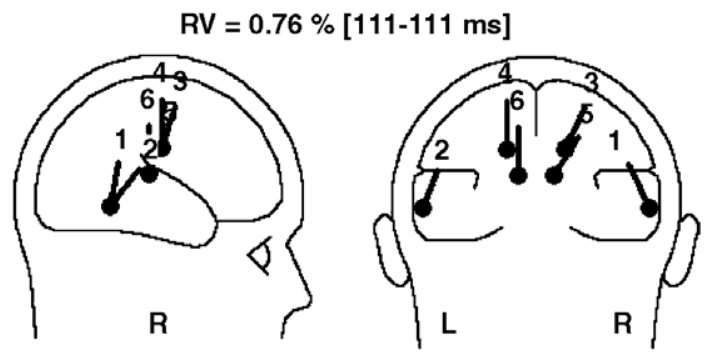

Fig. 7. The sources that contribute to the generation of the N100 ERP, at the moment of maximum (mean) N100 amplitude (111 ms) as a result of an auditory lead stimulus. It appears that 3 paired sources are involved.

modalities (mean $x y z$ coordinates of auditory resp. visual modality: $-9.6,32.9,77.3$ and-10.3, 28.7, 79.9).

\subsection{2. $N 100$}

Dipole analysis of the grand average ERPs elicited by $\mathrm{S} 1$ alone resulted in a six-source model (3 paired) for the auditory condition. The residual variance in this model reached a value of $0.76 \%$ (Fig. 7).

\section{Discussion}

To our knowledge, this is the first human study in which intra-modal and cross-modal sensory gating and sensorimotor gating were integrated into one experiment, thereby ruling out nonspecific differences between the two paradigms. P50 suppression was found following both auditory and visual conditioning stimuli in the 500-ms ISI only, with no significant difference between the modalities. Significant PPI was found with the auditory prepulse only, although roughly half of the subject population did show PPI to the visual prepulse while the other half did not. Dipole analysis of the P50 resulted in a three-source model for the auditory lead stimuli and a four-source model for the visual lead stimuli, of which one source appeared to be present in both modalities. The three-dimensional location parameters of this source were indicative of a frontal cortical site.

P50 suppression was found following both auditory and visual lead stimuli with an ISI of $500 \mathrm{~ms}$ only, in ratios that did not differ significantly from each other. In a complex study with healthy volunteers in which a tactile prestimulus was paired with an acoustic startling stimulus with ISIs of 60 and $360 \mathrm{~ms}$, P50 suppression was found in the 360-ms ISI only (Perlstein et al., 2001). The data of the Perlstein et al. (2001) study are consistent with the P50 suppression data of the present study, indicating that P50 suppression occurs with both intra-modal and cross-modal pairs of stimuli. Consequently, the inhibitory processes that regulate P50 suppression are not located in sensory cortical structures, such as the auditory or visual cortex. Furthermore, similar to the present study, Perlstein et al. (2001) did not find P50 suppression in their shortest ISI conditions (100 ms and $60 \mathrm{~ms}$, respectively), although this result was not confirmed in the studies by Nagamoto et al. $(1991,1996)$ using an ISI of $100 \mathrm{~ms}$. The fact that in the present study suppression was found exclusively in the longer ISI suggests that P50 suppression is not based on an intrinsic refractory mechanism.

Dipole analysis of the P50 ERP elicited by both auditory and visual lead stimuli led to models in which three and four sources, respectively, appeared to be active. One of these sources appeared to be present in both models: a source with frontal cortical origin. This observation is consistent with the data of Weisser et al. (2001), who found a source model for the auditory P50 with a remarkable similarity to the one found in the present study. In addition, in the study of Judd et al. (1992), patients with schizophrenia showed P50 suppression deficits at frontal, central and parietal electrode sites, with a tendency for the deficit to be most prominent in frontal electrode sites. Furthermore, in two studies of humans with brain damage, subjects with lesions in the prefrontal cortex showed an increase in distraction to irrelevant sensory information (Chao and Knight, 1995; Knight et al., 1995).

Recently, Adler et al. (1998) proposed a neurobiological model for sensory gating, in which the CA3 region of the hippocampus is indicated as both the origin of P50 suppression and the source of the P50 ERP as measured on the scalp. However, the frontal position of the source for the P50 ERP as found in the present study and in the study of Weisser et al. (2001) makes it unlikely that the hippocampal CA3 region is responsible for the P50 as measured on the scalp. Alternatively, the frontal location as source of the P50 potential might represent the frontal area innervated by the efferent fibers from the CA3 region, as mentioned in the study of Adler et al. (1998).

The acoustic lead stimulus not only reduced the P50 to the subsequent test stimulus, it also reduced longerlatency processing of the test stimulus, as indicated by the reduction in N100 amplitude, irrespective of ISI. This finding implies that the N100 amplitude can be reduced independently of the P50 amplitude, because no P50 suppression was found in the auditory ISI 100-ms condition in which N100 suppression was observed. This notion is strengthened by the fact that the visual 
lead stimulus was found to reduce the P50 response to the test stimulus, without affecting the N100 response.

The N100 amplitude was reduced only by intramodal pairs of auditory stimuli, regardless of ISI, which might indicate refractory processes. This result is consistent with the early study of Davis et al. (1966), who reported on the recovery processes involved in the N100 ERP. Later, Budd et al. (1998) showed that the decrement in response of the N100 is more consistent with refractory processes than with habituation to the presented stimuli. Furthermore, based on the pattern of changes in scalp topography, they argued that the sensitivity of the N100 to different ISIs suggests at least two generators of the N100 with different refractory properties. Taken together, the results indicate that P50 suppression and N100 suppression are independent phenomena, and that P50 suppression, in contrast to N100 suppression, is not based on intrinsic refractory mechanisms.

Dipole analysis of the N100 ERP elicited by auditory lead stimuli led to a model in which three paired sources appeared to be active. This result is consistent with the study of Scherg and Picton (1991), who also found a model for the auditory elicited N100 ERP based on three paired sources, similar to the model found in the present study. The absence of the frontocentral source of the P50 model in the N100 model supports the results of the amplitude data, in showing that the two ERPs are unrelated.

To our knowledge, this is the first time that PPI and P50 suppression were assessed in a fully integrated paradigm, in which an identical lead stimulus was used as the prepulse in the PPI trials and as the conditioning and testing stimulus in the P50 suppression trials. The results show that this same prepulse is not only capable of inducing P50 suppression but can also induce high levels of PPI. In contrast to the P50 suppression data, a higher level of PPI was found with the 100-ms ISI than with the 500-ms ISI. Graham (1975), used four different ISIs: $30,60,120$ and $240 \mathrm{~ms}$, of which the $120-\mathrm{ms}$ interval yielded the highest PPI, a result that was replicated subsequently (Braff et al., 1978; Dawson et al., 1995). These results are confirmed in the present study, and indicate that PPI and P50 suppression work on a different time basis: PPI is stronger with shorter ISIs, while P50 suppression becomes stronger with longer ISIs. Similar to P50 suppression, a number of studies exist in which cross-modal PPI was found, in both human (e.g.: Braff et al., 1992; Rossi et al., 1995; Perlstein et al., 2001) and animal studies (e.g.: Geyer et al., 1990; Campeau and Davis, 1995; Taylor et al., 1995; Ralph et al., 2001). This suggests that having the same modality for both the lead and startle stimuli is not a prerequisite for PPI. This conclusion is in part consistent with the present study, in which a visual lead stimulus induced PPI of acoustic startle stimuli in approximately half of our subject population. Remarkably, the half of the subject population that did show PPI to the visual lead stimulus had significantly higher P50 amplitudes to this stimulus than the other half. This effect could not be attributed to a difference between the groups in attending to the visual lead stimulus, because both groups were similar in P100 amplitude at electrode Oz, which is a measure for perception of visual stimuli. This result suggests that a minimum P50 amplitude to lead stimuli is essential for PPI to occur, at least for visual lead stimuli. Similarly, in the auditory condition, subjects with relatively high P50 amplitudes to the auditory lead stimulus appeared to exhibit higher PPI than subjects with lower amplitudes, although this difference did not reach significance with the current sample size. If confirmed, such a finding might indicate that a minimum P50 response to an auditory lead stimulus might be necessary for PPI to occur, as was seen in the visual condition. Consistent with earlier studies on the relationship between PPI and P50 suppression (Schwarzkopf et al., 1993; Oranje et al., 1999; Cadenhead et al., 2002), no correlations were found in the current study between these two gating measures, in either the auditory or visual modality. These observations further emphasize that the so-called sensory gating P50 and sensorimotor gating PPI measures reflect different underlying phenomena. Despite the fact that both measures were intended to assess the gating construct and both are reduced in patients with schizophrenia, the two measures are nonedundant and fundamentally different. In a study on PPI and glucose metabolism by Hazlett et al. (1998), the relative glucose metabolic rate (rGMR) in the frontal lobes of healthy volunteers correlated negatively with the amount of PPI, indicating that higher PPI was associated with increased activity in the frontal lobes. In this same study, unmedicated patients with schizophrenia showed less PPI than healthy controls and no correlation between rGMR and PPI. This study (Hazlett et al., 1998), together with the proposed frontal source of origin for the P50 as derived in the present study, seems to confirm the suggestion that the functional integrity of the prefrontal cortex is important for (though not essential for) sensorimotor gating (PPI) as well as for sensory gating (P50 suppression).

To conclude: In the auditory and visual conditions, respectively, two and three modality-specific and one modality-nonspecific sources for the P50 were found. Furthermore, no significant difference in the three-di- 
mensional coordinates of this modality-nonspecific source (frontally located) between the auditory and visual condition of the paradigm was found, suggesting that the models have this source in common. In addition, P50 suppression appears to be modality nonspecific and not based on refractory processes, both in contrast to suppression of the N100 amplitude, which indicates that suppression of the P50 and the N100 amplitudes are unrelated processes. Furthermore, the results revealed that PPI of an auditory loud stimulus following a visual lead stimulus requires a minimum P50 amplitude to the visual lead stimulus.

\section{Acknowledgment}

This study was initiated while M.A. Geyer was the F.C. Donders Chair at the University of Utrecht, the Netherlands. Furthermore, the study was supported by the U.S. National Institute of Mental Health grant MH42228.

\section{References}

Adler, L.E., Pachtman, E., Franks, R.D., Pecevich, M., Waldo, M.C., Freedman, R., 1982. Neurophysiological evidence for a defect in neuronal mechanisms involved in sensory gating in schizophrenia. Biological Psychiatry 17, 639-654.

Adler, L.E., Olincy, A., Waldo, M., Harris, J.G., Griffith, J., Stevens, K., Flach, K., Nagamoto, H., Bickford, P.C., Leonard, S., Freedman, R., 1998. Schizophrenia, sensory gating, and nicotinic receptors. Schizophrenia Bulletin 24, 189-202.

Andreasen, N.C., Flaum, M., Arndt, S., 1992. The Comprehensive Assessment of Symptoms and History (CASH): an instrument for assessing psychopathology and diagnosis. Archives of General Psychiatry 49, 615-623.

Bickford-Wimer, P.C., Nagamoto, H., Johnson, R., Adler, L.E., 1990. Auditory sensory gating in hippocampal neurons: a model system in the rat. Biological Psychiatry 27, 183-192.

Braff, D.L., Geyer, M.A., 1990. Sensorimotor gating and schizophrenia: human and animal model studies. Archives of General Psychiatry $47,181-188$.

Braff, D.L., Stone, C., Callaway, E., Geyer, M.A., Glick, I., Bali, L., 1978. Prestimulus effects on human startle reflex in normals and schizophrenics. Psychophysiology 15, 339-343.

Braff, D.L., Grillon, C., Geyer, M.A., 1992. Gating and habituation of the startle reflex in schizophrenic patients. Archives of General Psychiatry 49, 206-215.

Braff, D.L., Geyer, M.A., Swerdlow, N.R., 2001. Human studies of prepulse inhibition of startle: normal subjects, patient groups, and pharmacological studies. Psychopharmacology 156, 234-258.

Budd, T.W., Barry, R.J., Gordon, E., Rennie, C., Michie, P.T., 1998. Decrement of the N1 auditory event-related potential with stimulus repetition: habituation vs. refractoriness. International Journal of Psychophysiology 31, 51-68.

Cadenhead, K.S., Light, G.A., Geyer, M.A., McDowell, J.E., Braff, D.L., 2002. Neurobiological measures of schizotypal personality disorder: defining an inhibitory endophenotype? The American Journal of Psychiatry 159, 869-871.

Campeau, S., Davis, M., 1995. Prepulse inhibition of the acoustic startle reflex using visual and auditory prepulses: disruption by apomorphine. Psychopharmacology 117, 267-274.

Chao, L.L., Knight, R.T., 1995. Human prefrontal lesions increase distractibility to irrelevant sensory inputs. Neuroreport 6, $1605-1610$.

Clementz, B.A., Geyer, M.A., Braff, D.L., 1997. P50 suppression among schizophrenia and normal comparison subjects: a methodological analysis. Biological Psychiatry 41, 1035-1044.

Clementz, B.A., Geyer, M.A., Braff, D.L., 1998. Multiple site evaluation of P50 suppression among schizophrenia and normal comparison subjects. Schizophrenia Research 30, 71-80.

Davis, H., Mast, T., Yoshie, N., Zerlin, S., 1966. The slow response of the human cortex to auditory stimuli: recovery process. Electroencephalography and Clinical Neurophysiology 21, $105-113$.

Davis, M., Gendelman, S.D., Tischler, M.D., Gendelman, P.M., 1982. A primary acoustic startle circuit: lesion and stimulation studies. Journal of Neuroscience 2, 791-805.

Dawson, M.E., Schell, A.M., Hazlett, E.A., Filion, D.L., Nuechterlein, K.H., 1995. Attention, startle eye-blink modification, and psychosis proneness. In: Raine, A.E., Lencz, T.E., Mednick, S.A.E. (Eds.), Schizotypal Personality. Cambridge University Press, New York, pp xv, 510.

Endicott, J., Spitzer, R.L., 1978. A diagnostic interview: the Schedule for Affective Disorders and Schizophrenia. Archives of General Psychiatry 35, 837-844.

Ford, J.M., Roth, W.T., Menon, V., Pfefferbaum, A., 1999. Failures of automatic and strategic processing in schizophrenia: comparisons of event-related brain potential and startle blink modification. Schizophrenia Research 37, 149-163.

Freedman, R., Waldo, MC., Bickford-Wimer, P.C., Nagamoto, H., 1991. Elementary neuronal dysfunctions in schizophrenia. Schizophrenia Research 4, 233-243.

Geyer, M.A., Swerdlow, N.R., Mansbach, R.S., Braff, D.L., 1990. Startle response models of sensorimotor gating and habituation deficits in schizophrenia. Brain Research Bulletin 25, 485-498.

Graham, F.K., 1975. The more or less startling effects of weak prestimulation. Psychophysiology 12, 238-248.

Hazlett, E.A., Buchsbaum, M.S., Haznedar, M.M., Singer, M.B., Germans, M.K., Schnur, D.B., Jimenez, E.A., Buchsbaum, B.R., Troyer, B.T., 1998. Prefrontal cortex glucose metabolism and startle eyeblink modification abnormalities in unmedicated schizophrenia patients. Psychophysiology 35, 186-198.

Ison, J.R., Hoffman, H.S., 1983. Reflex modification in the domain of startle: II. The anomalous history of a robust and ubiquitous phenomenon. Psychological Bulletin 94, 3-17.

Judd, L.L., McAdams, L., Budnick, B., Braff, D.L., 1992. Senosory gating deficits in schizophrenia: new results. American Journal of Psychiatry 149, 488-493.

Kenemans, J.L., Molenaar, P.C., Verbaten, M.N., Slangen, J.L., 1991. Removal of the ocular artifact from the EEG: a comparison of time and frequency domain methods with simulated and real data. Psychophysiology 28, 114-121.

Knight, R.T., Grabowecky, M.F., Scabini, D., 1995. Role of human prefrontal cortex in attention control. In: Jasper, H.H., Riggio, S., Goldman-Rakic, P.S. (Eds.), Epilepsy and the Functional Anatomy of the Frontal Lobe. Raven Press, New York, pp. 21-36.

Koch, M., 1999. The neurobiology of startle. Progress in Neurobiology 59, 107-128. 
Kumari, V., Aasen, I., Sharma, T., 2004. Sex differences in prepulse inhibition deficits in chronic schizophrenia. Schizophrenia Research 69, 219-235.

McGhie, A., Chapman, J., 1961. Disorders of attention and perception in early schizophrenia. British Journal of Medical Psychology 34, $103-116$.

Nagamoto, H.T., Adler, L.E., Waldo, M.C., Freedman, R., 1989. Sensory gating in schizophrenics and normal controls: effects of changing stimulation interval. Biological Psychiatry 25, 549-561.

Nagamoto, H.T., Adler, L.E., Waldo, M.C., Griffith, J., 1991. Gating of auditory response in schizophrenics and normal control: effects of recording site and stimulation interval on the P50 wave. Schizophrenia Research 4, 31-40.

Nagamoto, H.T., Adler, L.E., Hea, R.A., Griffith, J.M., McRae, K.A., Freedman, R., 1996. Gating of auditory P50 in schizophrenics: unique effects of clozapine. Biological Psychiatry 40, 181-188.

Oranje, B., van Berckel, B.N., Kemner, C., van Ree, J.M., Kahn, R.S., Verbaten, M.N., 1999. P50 suppression and prepulse inhibition of the startle reflex in humans: a correlational study. Biological Psychiatry 45, 883-890.

Perlstein, W.M., Simons, R.F., Graham, F.K., 2001. Prepulse effects as a function of cortical projection system. Biological Psychology 56, 83-111.

Ralph, R.J., Paulus, M.P., Geyer, M.A., 2001. Strain-specific effects of amphetamine on prepulse inhibition and patterns of locomotor behavior in mice. Journal of Pharmacology and Experimental Therapeutics 298, 148-155.

Rossi, B., Vista, M., Farnetani, W., Gabrielli, L., Vignocchi, G., Bianchi, F., Berton, F., Francesconi, W., 1995. Modulation of electrically elicited blink reflex components by visual and acoustic prestimuli in man. International Journal of Psychophysiology 20, $177-187$.

Scherg, M., 1990. Fundamentals of dipole source potential analysis. In: Grandori, F., Hoke, M., Romani, G.L. (Eds.), Auditory Evoked Magnetic Fields and Electric Potentials. Karger, Basel, pp. 40-69.
Scherg, M., Picton, T.W., 1991. Separation and identification of event-related potential components by brain electric source analysis. In: Brunia, C.H., Mulder, G., Verbaten, M.N. (Eds.), Event related Brain Research (EEG Suppl. 42). Elsevier Science Publishers, B.V., Amsterdam, pp. 24-37.

Schwarzkopf, S.B., Lamberti, J.S., Smith, D.A., 1993. Concurrent assessment of acoustic startle and auditory P50 evoked potential measures of sensory inhibition. Biological Psychiatry 33, $815-828$.

Swerdlow, N.R., Caine, S.B., Braff, D.L., Geyer, M.A., 1992. The neural substrates of sensorimotor gating of the startle reflex: a review of recent findings and their implications. Journal of Psychopharmacology 6, 176-190.

Swerdlow, N.R., Geyer, M.A., Braff, D.L., 2001. Neural circuit regulation of prepulse inhibition of startle in the rat: current knowledge and future challenges. Psychopharmacology 156, 194-215.

Taylor, M.K., Ison, J.R., Schwarzkopf, S.B., 1995. Effects of single and repeated exposure to apomorphine on the acoustic startle reflex and its inhibition by a visual prepulse. Psychopharmacology $120,117-127$.

Venables, P.H., 1964. Input dysfunction in schizophrenia. In: Maher, B.A. (Ed.), Progress in Experimental Personality Research. Academic Press, New York, pp. 1-47.

Ward, P.B., Hoffer, L.D., Liebert, B.J., Catts, S.V., O’Donnell, M., Adler, L.E., 1996. Replication of a P50 auditory gating deficit in Australian patients with schizophrenia. Psychiatry Research 64, $121-135$.

Weisser, R., Weisbrod, M., Roehrig, M., Rupp, A., Schroeder, J., Scherg, M., 2001. Is frontal lobe involved in the generation of auditory evoked P50? Neuroreport 12, 3303-3307.

Wynn, J.K., Dawson, M.E., Schell, A.M., McGee, M., Salveson, D., Green, M.F., 2004. Prepulse facilitation and prepulse inhibition in schizophrenia patients and their unaffected siblings. Biological Psychiatry 55, 518-523. 